\section{Accuracy in the Assessment of Cough Peak Flow: Good Progress for a "Work in Progress"}

\section{To the Editor:}

The measurement of cough peak flow $(\mathrm{CPF})$ is becoming more prominent and established in the literature, and new evidence confirms its use in research and clinical practice. In a recent study, Del Amo Castrillo and colleagues ${ }^{1}$ compared 2 cough augmentation modalities using a home ventilator in patients with neuromuscular disease. The authors used CPF as an outcome variable to evaluate the relative effectiveness of air stacking versus volumetric cough mode; they applied a threshold of CPF $<270 \mathrm{~L} / \mathrm{min}$ to identify individuals with ineffective cough. ${ }^{1}$ In 2018, Respiratory Care published the work of Rose and colleagues, ${ }^{2}$ who surveyed 155 Canadian and British respiratory clinicians about the adoption of guideline recommendations. In this study, $\mathrm{CPF}$ was the most commonly reported method for assessing cough effectiveness ( $87 \%$ of respondents); $>80 \%$ of respondents reported that they assessed CPF before initiating treatments for airway clearance. $^{2}$

The use of CPF threshold values has been described in several clinical practice guidelines, with the aim to assist clinical decision-making. For example, the Canadian Thoracic Society guideline on home mechanical ventilation suggests the need for assisted airway clearance if CPF drops below the $270 \mathrm{~L} / \mathrm{min}$ threshold in at-risk patients. ${ }^{3}$ Similarly, the British Thoracic Society guideline for the physiotherapy management of the adult medical patient describes CPF thresholds of 270 and $160 \mathrm{~L} / \mathrm{min}$, with escalating recommendations for assisted airway clearance in patients with neuromuscular disease. $^{4}$

Correspondence: Stefan Tino Kulnik PhD MRes PT, Faculty of Health, Social Care and Education, Kingston University, and St George's, University of London, 6th Floor, Hunter Wing, Bay 19/Desk 15, St George's Campus, Cranmer Terrace, London SW17 0RE, United Kingdom. E-mail: s.t.kulnik@sgul.kingston.ac.uk.

The authors have disclosed no conflicts.

DOI: $10.4187 /$ respcare. 07454
Earlier this year, the European Respiratory Society (ERS) statement on respiratory muscle testing provided high-profile guidance on the measurement of $\mathrm{CPF}{ }^{5}$ In contrast to its preceding American Thoracic Society (ATS)/ERS statement, ${ }^{6}$ section 1.2.3 of the new statement includes, for the first time, much needed guidance to improve the standardization of $\mathrm{CPF}$ measurements, such as the preferred testing position, suggested number of repetitions, and target variability. ${ }^{5}$ Importantly, the document also highlights potential differences in absolute values of $\mathrm{CPF}$ when different measurement devices are used, such as pneumotachograph-based devices as opposed to peak flow meters with a sliding marker. For researchers and clinicians with an interest in neuromuscular weakness of cough and effective clearance of airway secretions, this is important information and a great step forward.

Although several studies have examined agreement between different devices for the measurement of peak flow during a forced maneuver, very few studies have investigated the same with respect to the measurement of CPF specifically. Sancho and colleagues ${ }^{7}$ compared CPF measured with a pneumotachograph spirometer against a peak flow meter with a sliding marker in subjects with neuromuscular disease. They reported Bland-Altman limits of agreement of $\pm 66 \mathrm{~L} / \mathrm{min}$, ie, the difference in measurements between the two devices was as large as $66 \mathrm{~L} / \mathrm{min}$ in either direction of the average value. Two of our group of co-authors (STK, VM) published a study in which we examined agreement between two types of pneumotachograph spirometers, two types of peak flow meters with sliding markers, and a laboratory pneumotachograph system in healthy subjects and using a mechanical cough simulator. ${ }^{8}$ Using the laboratory pneumotachograph as the accepted standard, Bland-Altman limits of agreement for the spirometers and peak flow meters spanned $150 \mathrm{~L} / \mathrm{min}^{8}$ Rodrigues and colleagues ${ }^{9}$ reported differences in CPF measurements in subjects with neuromuscular disease. Comparing values obtained with a pneumotachograph spirometer against a peak flow meter, they described BlandAltman limits of agreement spanning $338 \mathrm{~L} / \mathrm{min} .{ }^{9}$ In a more recent study in subjects with Duchenne muscular dystrophy, Kikuchi and colleagues ${ }^{10}$ compared several cough-augmentation methods and observed differences in CPF readings depending on the measurement device used. The authors reported differences in measurements between a spirometer and a mechanical insufflation-exsufflation device and between a peak flow meter and a spirometer, with Bland-Altman limits of agreement spanning $50 \mathrm{~L} / \mathrm{min}^{10}$

These studies and the ERS statement highlight an important current limitation in the measurement of $\mathrm{CPF}$, which is that different measurement devices perform variably, leading to potentially substantial inaccuracy in CPF measurements. Researchers and clinicians need to be aware of this, particularly when using absolute values of $\mathrm{CPF}$ as thresholds to guide clinical decisions. Due to the wide limits of agreement, it is possible that one patient's CPF value may lie on either side of a proposed threshold, depending on the device used. This could lead to different treatment recommendations.

In his editorial on the study by Del Amo Castrillo et al, ${ }^{1}$ Michel Toussaint ${ }^{11}$ reminds us of the need for strong and objective indicators of effective cough. Although we have moved forward as a community and are recognizing the potential of $\mathrm{CPF}$ for our clinical practice, we need to continue to work toward agreed standardization and appropriate implementation so that $\mathrm{CPF}$ can become a reliable and meaningful clinical tool. Further research should explore reasons for variations between flow measurement devices and possible solutions. One hypothesis for the observed inaccuracies is that the unpredictable dynamics of turbulent flow during cough have a greater impact on flow measurement than the more predictable dynamics of laminar flow during a forced spirometric maneuver with open glottis. ${ }^{8}$ Due to its dynamic nature, the mathematical modelling and prediction of turbulent flow is challenging. It might therefore not be possible to achieve good agreement of CPF measurements across different devices through corrective formulae. A pragmatic approach could be to agree on the use of one particular type of instrument, both for the identification and validation of CPF clinical threshold values in research studies and for the measurement of CPF in clinical practice; a drawback to this suggestion is the unfair commercial advantage for one particular 


\section{CORRESPONDENCE}

manufacturer that this approach may create.

Another hypothesis to explain inaccuracy in CPF measurements is that the frequency response of devices is inadequate. Frequency response, in simple terms, describes the consistency between input and output. Peak flow meters and spirometers are designed and calibrated to meet the needs of spirometric maneuvers, which have a slower rise time (ie, time from zero flow to peak flow) than a cough. The much shorter rise time (input) in cough combined with inadequate frequency response of devices may account for some of the observed inaccuracy. ${ }^{8}$ Manufacturers could support clinicians and researchers by characterizing their devices more fully and declaring whether devices are suitable for the measurement of CPF based on the frequency response. Greater critical emphasis on the clinical relevance of CPF could provide impetus for manufacturers to develop instruments that adequately meet the performance criteria for accurate $\mathrm{CPF}$ measurement.

In conclusion, although CPF could be a useful parameter to guide our clinical decision making, we should be cautious about the devices we use and the accuracy of our CPF measurements. As it stands, the accuracy of $\mathrm{CPF}$ measurements presents a challenge for further research. We hope that our letter will contribute to highlighting this important issue and will support colleagues in the interpretation of study findings and clinical guidelines. Progress in this work is the way forward to benefit our patients.

\section{Stefan Tino Kulnik \\ Agnieszka Lewko}

Faculty of Health, Social Care and Education Kingston University and

St George's University of London London, United Kingdom

Victoria MacBean

Centre for Human Performance, Exercise and Rehabilitation

Brunel University London London, United Kingdom

Arietta Spinou

Population Health Sciences

Faculty of Life Sciences and Medicine

King's College London

London, United Kingdom

\section{REFERENCES}

1. Del Amo Castrillo L, Lacombe M, Boré A, Vaugier I, Falaize L, Orlikowski D, et al. Comparison of two cough-augmentation techniques delivered by home ventilator in subjects with neuromuscular disease. Respir Care 2019;64(3):255-261.

2. Rose L, McKim D, Leasa D, Nonoyama M, Tandon A, Kaminska M, et al. Monitoring cough effectiveness and use of airway clearance strategies: a Canadian and UK survey. Respir Care 2018;63(12):15061513.

3. McKim DA, Road J, Avendano M, Abdool $\mathrm{S}$, Cote F, Duguid N, et al. Home mechanical ventilation: a Canadian Thoracic Society clinical practice guideline. Can Respir J 2011;18(4):197-215
4. Bott J, Blumenthal S, Buxton M, Ellum S, Falconer C, Garrod R, et al. Guidelines for the physiotherapy management of the adult, medical, spontaneously breathing patient. Thorax 2009;64(Suppl 1):i1-i51.

5. Laveneziana $P$, Albuquerque A, Aliverti A, Babb T, Barreiro E, Dres M, et al. ERS statement on respiratory muscle testing at rest and during exercise. Eur Respir J 2019;53(6):1801214.

6. American Thoracic Society/European Respiratory Society. ATS/ERS Statement on respiratory muscle testing. Am J Respir Crit Care Med 2002;166(4):518-624.

7. Sancho J, Servera E, Díaz J, Marín J. Comparison of peak cough flows measured by pneumotachograph and a portable peak flow meter. Am J Phys Med Rehabil 2004; 83(8):608-612.

8. Kulnik ST, MacBean V, Birring SS, Moxham J, Rafferty GF, Kalra L. Accuracy of portable devices in measuring peak cough flow. Physiol Meas 2015;36(2):243257.

9. Rodrigues PR, Brito PU, Fernandes L, Rodrigues C, Reis A, Moita J. Peak cough flow measurement with a pneumotachograph and a portable peak flow meter in patients with neuromuscular diseases. Rev Port Pneumol (2006) 2017;23(1): 39-42.

10. Kikuchi K, Satake M, Kimoto Y, Iwasawa S, Suzuki R, Kobayashi M, et al. Approaches to cough peak flow measurement with Duchenne muscular dystrophy. Respir Care 2018;63(12):1514-1519.

11. Toussaint M. Augmenting cough via home ventilators in subjects with neuromuscular disease: simple, effective, and clever. Respir Care 2019;64(3):355-357. 